Bull. Austral. Math. Soc.

30E10, 41A20

VOL. $38(1988) \quad$ [415-422]

\title{
ON CERTAIN SEQUENCES OF LEAST SQUARES APPROXIMANTS
}

\author{
M.A. BOKHARI
}

\begin{abstract}
A sequence of certain rational functions is determined where each member solves an $\ell_{2}$ minimisation problem on a "large" set of roots of unity. The sequence is compared with another sequence of $L_{2}$-rational approximants. Our main result extends a result of Rivlin on Walsh type equiconvergence.
\end{abstract}

\section{INTRODUCTION}

Let $A_{\rho}, 1<\rho<\infty$, be the set of functions $f(z)$ analytic in $|z|<\rho$ and having a singularity on the circle $|z|=\rho$. Let $\pi_{n}$ denote the class of all polynomials of degree $\leqslant n$. If $f(z)=\sum_{j=0}^{\infty} a_{j} z^{j}$, then we put

$$
S_{n-1}(z, f)=\sum_{j=0}^{n-1} a_{j} z^{j}
$$

Let $p_{n-1, m}(z, f), m \geqslant n$, denote the polynomial of degree $n-1$ of least square approximation to $f(z)$ on the $m$ th roots of unity. Then an extension of a result of Walsh $[3$, p. 153] due to Rivlin [1, Theorem 1] can be stated as follows:

Theorem. Let $q$ be a fixed positive integer and $m=q n+c$ with $0 \leqslant c \leqslant q-1$. If $f \in A_{\rho}, 1<\rho<\infty$ then,

$$
\lim _{n \rightarrow \infty}\left\{p_{n-1, m}(z, f)-S_{n-1}(z, f)\right\}=0, \quad|z|<\rho^{q+1}
$$

the convergence being uniform and geometric in $|z| \leqslant r<\rho^{q+1}$. Moreover, the result is sharp in the sense that (1.2) fails for every $z$ satisfying $|z|=\rho^{q+1}$ for any $f \in A_{\rho}$.

Recently, Saff and Sharma [2] discussed the behaviour of certain sequences of rational interpolants. Walsh's theorem [3, p. 153] is again a special case of their result. In the present paper we discuss equiconvergence of certain rational sequences of the form used by Saff and Sharma [2].

Received 30 July, 1987

The author is grateful to Professors A. Sharma and E.B. Saff for letting him use their earlier notes on this Problem.

Copyright Clearance Centre, Inc. Serial-fee code: 0004-9729/88 \$A2.00+0.00. 
Section 2 deals with the $\ell_{2}$-minimisation problem on the set $\left\{\omega^{k}\right\}_{0}^{g n-1}$, where $\omega$ is a primitive $(q n)$ th root of unity. Its solution will replace the polynomial $p_{n-1, m}(z, f)$ in (1.2) as stated in our main result (see Theorem 2.1). Similarly, in place of the Taylor polynomial $S_{n-1}(z, f)$ in (1.2), we shall consider the unique rational function $r_{n+m, n}(z, f)[2,(1.4)]$ where

$$
r_{n+m, n}(z, f)=\frac{P_{n+m, n}(z, f)}{z^{n}-\sigma^{n}}, P_{n+m, n}(z, f) \in \pi_{n+m}, m \geqslant-1, \sigma>1,
$$

which minimises the integral

$$
\int_{|z|=1}|f(z)-r(z)|^{2}|d z|
$$

over all rational functions of the form $r(z)=\frac{p(z)}{z^{n}-\sigma^{n}}, p(z) \in \pi_{n+m}$. The minimisation problem will be solved in Section 3. We shall prove our main result in Section 4.

\section{A MiNimisation PROBLEM AND STATEMENT OF RESUlT}

Consider the following problem:

(P1). Let $m \geqslant-1$ and $q \geqslant 2$ be fixed integers and let $\omega=\exp (2 \pi i / q n)$. For $f \in A_{\rho}$, we want to minimise

$$
\sum_{k=0}^{q n-1}\left|f\left(\omega^{k}\right)-R\left(\omega^{k}, f\right)\right|^{2}
$$

over all ratioinal functions of the form

$$
R(z, f)=\frac{p(z)}{z^{n}-\sigma^{n}}, \quad p(z) \in \pi_{n+m} .
$$

If the solution of the problem (P1) is given by

$$
R_{n+m, n}^{*}(z, f)=\frac{P_{n+m, n}^{*}(z, f)}{z^{n}-\sigma^{n}}, \quad P_{n+m, n}^{*}(z, f) \in \pi_{n+m},
$$

then we can state our main result as:

Theorem 2.1. Let $m \geqslant-1$ and $q \geqslant 2$ be two fixed integers and let $\sigma>1$. If $f \in A_{\rho}, 1<\rho<\infty$, then

$$
\lim _{n \rightarrow \infty}\left\{R_{n+m, n}^{*}(z, f)-r_{n+m, n}(z, f)\right\}=0 \begin{cases}|z|<\rho^{1+q} & \text { if } \sigma \geqslant \rho^{1+q}, \\ |z| \neq \sigma & \text { if } \sigma<\rho^{1+q},\end{cases}
$$

the convergence being uniform and geometric in any compact subset of the regions described above. Moreover, the result is sharp in the sense that for each $|z|=\rho^{1+q}$ with $\sigma \geqslant \rho^{1+q}$, there is an $\hat{f} \in A_{\rho}$ for which (2.3) does not hold.

Proof of the preceding theorem will be given in Section 4. First we turn to the reformulation of the minimisation problem (P1) which requires the following lemma: 
LEMMA 2.1. Let $m \geqslant-1$ and $q \geqslant 2$ be fixed integers and let $g(z)=$ $P(z) /\left(z^{n}-\sigma^{n}\right)$, where $P(z)=\sum_{j=0}^{n+m} c_{j} z^{j} \in \pi_{n+m}$ is given. Then the Lagrange interpolant of $g(z)$ on the $(q n)$ th roots of unity is given by

$$
L_{q n-1}(z, g)=\sum_{\nu=0}^{q n-1} A_{\nu} z^{\nu}
$$

where

$$
A_{\nu n+j}= \begin{cases}\lambda_{1} c_{j}+\lambda_{q} c_{j+n}, & \nu=0, \quad 0 \leqslant j \leqslant m \\ \lambda_{\nu+1} c_{j}, & 0 \leqslant \nu \leqslant q-1, \quad m+1 \leqslant j \leqslant n-1 \\ \lambda_{\nu+1} c_{j}+\lambda_{\nu} c_{j+n}, & 1 \leqslant \nu \leqslant q-1, \quad 0 \leqslant j \leqslant m\end{cases}
$$

with

$$
\lambda_{\nu}=\sigma^{(q-\nu) n} /\left(1-\sigma^{q n}\right), \quad \nu=1,2, \ldots, q
$$

PROOF: The Lagrange interpolant of the function $\left(z^{n}-\sigma^{n}\right)^{-1}$ on the $(q n)$ th roots of unity is given by

$$
L_{q n-1}\left(z,\left(z^{n}-\sigma^{n}\right)^{-1}\right)=\frac{z^{q n}-\sigma^{q n}}{\left(z^{n}-\sigma^{n}\right)\left(1-\sigma^{q^{n}}\right)}=\sum_{\nu=1}^{q} \lambda_{\nu} z^{(\nu-1) n}
$$

For $z=\omega^{k}$, we have

$$
\left(\omega^{k n}-\sigma^{n}\right)^{-1}=\sum_{\nu=1}^{q} \lambda_{\nu} \omega^{(\nu-1) n k}, \quad k=0,1,2, \ldots, q n-1
$$

If we multiply (2.6) by $P\left(\omega^{k}\right)=\sum_{j=0}^{n+m} c_{j} \omega^{k j}$ then a simple calculation leads to

$$
\begin{aligned}
\frac{P\left(\omega^{k}\right)}{\omega^{k n}-\sigma^{n}} & =\lambda_{1} \sum_{j=0}^{n+m} c_{j} \omega^{k j} \\
& +\lambda_{2} \sum_{j=n}^{2 n+m} c_{j-n} \omega^{k j}+\ldots+\lambda_{q} \sum_{j=(q-1) n}^{q n+m} c_{j-(q-1) n} \omega^{k j}
\end{aligned}
$$

Notice that in the above, each of the $q$ summations has $m+1$ distinct powers of $\omega^{k}$ which also appear in the preceding sum. If we group the terms involving identical 
powers of $\omega^{k}$ in separate summations and then rearrange them, we obtain for each $k=0,1,2, \ldots, q n-1$,

$$
\begin{aligned}
\frac{P\left(\omega^{k}\right)}{\omega^{k n}-\sigma^{n}} & =\sum_{j=0}^{m}\left[\lambda_{1} c_{j}+\lambda_{q} c_{j+n}\right] \omega^{j k}+\sum_{\nu=0}^{q-1} \sum_{j=m+1}^{n-1} \lambda_{\nu+1} c_{j} \omega^{(\nu n+j) k} \\
& +\sum_{\nu=1}^{q-1} \sum_{j=0}^{m}\left[\lambda_{\nu+1} c_{j}+\lambda_{\nu} c_{j+n}\right] \omega^{(\nu n+j) k}
\end{aligned}
$$

which reduces to $L_{q n-1}\left(\omega^{k}, g\right)=\sum_{j=0}^{q n-1} A_{j} \omega^{j k}$ on recalling that $\omega$ is a primitive $(q n)$ th root of unity, where the $A_{j}$ 's are given by (2.4).

REMARK 2.1: If the $a_{\nu}$ 's are given complex numbers, then from the properties of the roots of unity, it follows that

$$
\sum_{k=0}^{q n-1}\left|\sum_{\nu=0}^{q n-1} a_{\nu} \omega^{k \nu}\right|^{2}=q n \sum_{\nu=0}^{q n-1}\left|a_{\nu}\right|^{2}
$$

REMARK 2.2: If $L_{q n-1}(z, f)=\sum_{j=0}^{q n-1} b_{j} z^{j}$ is the Lagrange interpolant of degree $q n-1$ to $f(z) \in A_{\rho}$ in the $(q n)$ th roots of unity, then we recall that

$$
b_{j}=\frac{1}{2 \pi i} \int_{\Gamma} \frac{f(t) t^{q^{n-j-1}}}{t^{q^{n}}-1} d t, \quad 0,1,2, \ldots, q n-1
$$

where $\Gamma$ is the circle $|t|=R, \quad 1<R<\rho$.

REMARK 2.3: Since $L_{q n-1}\left(\omega^{k}, f\right)=\sum_{\nu=0}^{q n-1} b_{\nu} \omega^{k \nu}=f\left(\omega^{k}\right)$ and $L_{q n-1}\left(\omega^{k}, g\right)=\sum_{\nu=0}^{q n-1} A_{\nu} \omega^{k \nu}, k=0,1,2, \ldots, q n-1$, it follows from $(2.7)$ that the minimisation problem (P1) is equivalent to the problem (P2):

(P2). Minimise

$$
G=\sum_{\nu=0}^{q n-1}\left|b_{\nu}-A_{\nu}\right|^{2}
$$

over the $c_{j}$ 's, $j=0,1,2, \ldots, n+m$, where the $A_{\nu}$ 's are given by (2.4).

It may be noted that the $b_{\nu}$ 's are well-defined from (2.8) and that the $A_{\nu}$ 's are functions of the $c_{j}$ 's. 


\section{Solution of the minimisation PRoblem (P1)}

The following proposition determines the solution of the problem (P1):

Proposition 2.1. The polynomial $P_{n+m, n}^{*}(z, f)$ (see (2.2)) which solves the minimisation problem $(P 1)$ is given by

$$
P_{n+m, n}^{*}(z, f)=\sum_{\nu=0}^{n+m} p_{\nu} z^{\nu}
$$

where

$$
p_{\nu^{\prime}}= \begin{cases}\frac{-b_{\nu} \sigma^{n}+\frac{\sigma^{(q-1) n}\left(1-\sigma^{2 n}\right)}{1-\sigma^{(q-1)^{2 n}}} \sum_{j=1}^{q-1} \sigma^{-j n} b_{\nu+j n},}{\sigma^{(q-1) n}\left(1-\sigma^{2 n}\right)} \sum_{j=0}^{q-1} \sigma^{-j n} b_{\nu+j n}, & 0 \leqslant \nu \leqslant m \\ b_{\nu-n}-\frac{\sigma^{2(q-1) n}\left(1-\sigma^{2 n}\right)}{1-\sigma^{(q-1)^{2 n}}} \sum_{j=1}^{q-1} \sigma^{-j n} b_{\nu+(j-1) n}, & n \leqslant \nu \leqslant n+m,\end{cases}
$$

and the $b_{\nu}, \nu=0,1, \ldots, q n-1$ are given by (2.8).

Proof: Since (P1) is equivalent to (P2), it is sufficient to solve the system of equations $\frac{\partial G}{\partial \widetilde{c}_{\nu}}=0, \nu=0,1,2, \ldots, n+m$, in terms of the $\lambda_{\nu}$ 's and $b_{j}$ 's. Using (2.4), we can rewrite $(2.9)$ as:

$$
\begin{aligned}
G & =\sum_{j=0}^{m}\left|b_{j}-\lambda_{1} c_{j}-\lambda_{q} c_{j+n}\right|^{2}+\sum_{\nu=0}^{q-1} \sum_{j=m+1}^{n-1}\left|b_{j+\nu n}-\lambda_{\nu+1} c_{j}\right|^{2} \\
& +\sum_{\nu=1}^{q-1} \sum_{j=0}^{m}\left|b_{j+\nu n}-\lambda_{\nu+1} c_{j}-\lambda_{\nu} c_{j+n}\right|^{2} .
\end{aligned}
$$

Therefore, for $0 \leqslant j \leqslant m$, we have

$$
\begin{aligned}
\frac{\partial G}{\partial \bar{c}_{j}} & =-\left(b_{j}-\lambda_{1} c_{j}-\lambda_{q} c_{j+n}\right) \lambda_{1}-\sum_{\nu=1}^{q-1}\left(b_{j+\nu n}-\lambda_{\nu+1} c_{j}-\lambda_{\nu} c_{j+n}\right) \lambda_{\nu+1} \\
& =\sum_{\nu=1}^{q} \lambda_{\nu}^{2} c_{j}+\left(\lambda_{q} \lambda_{1}+\lambda_{1} \lambda_{2}+\cdots+\lambda_{q-1} \lambda_{q}\right) c_{j+n}-\sum_{\nu=0}^{q-1} \lambda_{\nu+1} b_{j+\nu n}
\end{aligned}
$$

and

$$
\begin{aligned}
& \frac{\partial G}{\partial \bar{c}_{n+j}}=-\left(b_{j}-\lambda_{1} c_{j}-\lambda_{q} c_{j+n}\right) \lambda_{q}-\sum_{\nu=1}^{q-1}\left(b_{j+\nu n}-\lambda_{\nu+1} c_{j}-\lambda_{\nu} c_{j+n}\right) \lambda_{\nu} \\
& =\sum_{\nu=1}^{q} \lambda_{\nu}^{2} c_{j+n}+\left(\lambda_{q} \lambda_{1}+\lambda_{1} \lambda_{2}+\cdots+\lambda_{q-1} \lambda_{q}\right) c_{j}-\lambda_{q} b_{j}-\sum_{\nu=1}^{q-1} \lambda_{\nu} b_{j+\nu n} .
\end{aligned}
$$


Also, for $m+1 \leqslant j \leqslant n-1$, we have

$$
\frac{\partial G}{\partial \bar{c}_{j}}=-\sum_{\nu=0}^{q-1}\left(b_{j+\nu n}-\lambda_{\nu+1} c_{j}\right) \lambda_{\nu+1}=\sum_{\nu=0}^{q-1} \lambda_{\nu+1}^{2} c_{j}-\sum_{\nu=0}^{q-1} \lambda_{\nu+1} b_{j+\nu n} .
$$

For the sake of simplicity, we set

$$
\left\{\begin{array}{l}
\alpha=\lambda_{1}^{2}+\lambda_{2}^{2}+\cdots+\lambda_{q}^{2}=\left(\frac{1+\sigma^{q n}}{1-\sigma^{2 n}}\right) \lambda_{q} \\
\beta=\lambda_{q} \lambda_{1}+\lambda_{1} \lambda_{2}+\cdots+\lambda_{q-1} \lambda_{q}=\left(\frac{\sigma^{n}+\sigma^{(q-1) n}}{1-\sigma^{2 n}}\right) \lambda_{q}
\end{array}\right.
$$

Then from (3.2)-(3.4) we obtain, for $j=0,1, \ldots, m$ :

$$
\begin{cases}\frac{\partial G}{\partial \bar{c}_{j}}=\alpha c_{j}+\beta c_{n+j}-\sum_{\nu=0}^{q-1} \lambda_{\nu+1} b_{\nu n+j}, & (0 \leqslant j \leqslant m) \\ \frac{\partial G}{\partial \bar{c}_{n+j}}=\alpha c_{n+j}+\beta c_{j}-\lambda_{q} b_{j}-\sum_{\nu=1}^{q-1} \lambda_{\nu} b_{\nu n+j}, & \end{cases}
$$

and for $m+1 \leqslant j \leqslant n-1$, we see that

$$
\frac{\partial G}{\partial \bar{c}_{j}}=\alpha c_{j}-\sum_{\nu=0}^{q-1} \lambda_{\nu+1} b_{\nu n+j}
$$

Setting the partial derivatives $\frac{\partial G}{\partial \bar{c}_{\nu}}$ equal to zero and then solving the system of equations so obtained simultaneously for $c_{\nu}$ and $c_{\nu+n}, \nu=0,1, \ldots, m$, we obtain

$$
c_{\nu}=\left(\alpha^{2}-\beta^{2}\right)^{-1}\left\{\alpha \sum_{j=0}^{q-1} \lambda_{j+1} b_{j n+\nu}-\beta \sum_{j=1}^{q-1} \lambda_{j} b_{j n+\nu}-\beta \lambda_{q} b_{\nu}\right\}
$$

and

$$
c_{n+\nu}=\left(\alpha^{2}-\beta^{2}\right)^{-1}\left\{\alpha \sum_{j=1}^{q-1} \lambda_{j} b_{j n+\nu}+\alpha \lambda_{q} b_{\nu}-\beta \sum_{j=0}^{q-1} \lambda_{j+1} b_{j n+\nu}\right\}
$$

Also from (3.7) we have

$$
c_{\nu}=\alpha^{-1} \sum_{j=1}^{q} \lambda_{j} b_{(j-1) n+\nu}, \quad m+1 \leqslant \nu \leqslant n-1 .
$$

We recall that $c_{\nu}, 0 \leqslant \nu \leqslant n+m$, as determined in (3.8)-(3.10), are the coefficients of the polynomial $P_{n+m, n}^{*}(z, f)$ (see (2.2)) which minimises the expression given by (2.1).

Finally, replacing $\alpha, \beta$ and $\lambda_{j}$ 's in $c_{\nu},(\nu=0,1, \ldots, n+m)$, by their respective values from (3.5) and (2.5) we obtain the relation (3.1) after some simple arithmetic.

REMARK 3.1: When $q=1$, the solution of the problem (P1) is not unique for all $m>-1$. However, $R_{n-1, n}^{*}(z, f)$ is uniquely defined for $q=1$. It turns out that $R_{n-1, n}^{*}(z, f)$, in this case, interpolates the function $f(z)$ in the $n$th roots of unity. 


\section{Proof of Theorem 2.1}

It is known [2] that an integral representation of the rational function $r_{n+m, n}(z, f)$ is given by

$$
r_{n+m, n}(z, f)=\frac{1}{2 \pi i} \int_{\Gamma} \frac{\left(t^{n}-\sigma^{n}\right) f(t)}{\left(z^{n}-\sigma^{n}\right)(t-z)} \sum_{j=1}^{3} A_{j}(t, z) d t
$$

where $\Gamma$ is the circle $|t|=R, 1<R<\rho$ and $A_{j}(t, z), j=1,2,3$ and given by

$$
\left\{\begin{array}{l}
A_{1}(t, z)=\frac{t^{m+1}-z^{m+1}}{t^{m+1}}, \quad A_{2}(t, z)=\frac{z^{m+1}\left(t^{n-m-1}-z^{n-m-1}\right)}{t^{n}-\sigma^{-1}} \\
A_{3}(t, z)=z^{n}\left(t^{m+1}-z^{m+1}\right) / t^{m+1}\left(t^{n}-\sigma^{-n}\right)
\end{array}\right.
$$

Also, from (2.2), (2.8) and (3.1), we obtain after some algebraic operations

$$
R_{n+m, n}^{*}(z, f)=\frac{1}{2 \pi i} \int_{\Gamma} \frac{f(t)}{\left(z^{n}-\sigma^{n}\right)(t-z)\left(t^{q^{n}}-1\right)} \sum_{j=1}^{3} A_{j}(t, z) B_{j}(t, \sigma) d t
$$

with

$$
\left\{\begin{array}{l}
B_{1}(t, \sigma)=\sigma^{-(q-2) n} B(t, \sigma)-t^{q n} \sigma^{n} \\
B_{2}(t, \sigma)=\left(t^{q n}-\sigma^{-q n}\right)\left(\sigma^{-n}-\sigma^{n}\right) /\left(1+\sigma^{-q n}\right) \\
B_{3}(t, \sigma)=\left(t^{n}-\sigma^{-n}\right)\left\{t^{g n}-\sigma^{n} B(t, \sigma)\right\}
\end{array}\right.
$$

where

$$
B(t, \sigma)=\frac{t^{n}\left(t^{(q-1) n}-\sigma^{-(q-1) n}\right)\left(1-\sigma^{-2 n}\right)}{\left(t^{n}-\sigma^{-n}\right)\left(1-\sigma^{-2(q-1) n}\right)}
$$

Therefore,

$$
R_{n+m, n}^{*}(z, f)-r_{n+m, n}(z, f)=\frac{1}{2 \pi i} \int_{\Gamma} \sum_{j=1}^{3} \frac{A_{j}(t, z) K_{j}(t, \sigma)}{\left(z^{n}-\sigma^{n}\right)(t-z)\left(t^{q^{n}}-1\right)} f(t) d t
$$

where $K_{j}(t, \sigma)=B_{j}(t, \sigma)-\left(t^{q n}-1\right)\left(t^{n}-\sigma^{n}\right), j=1,2,3$ can be explicitly rewritten after some simplification as

$$
\left\{\begin{array}{l}
K_{1}(t, \sigma)=\sigma^{-(q-2) n} B(t, \sigma)-t^{(q+1) n}+t^{n}-\sigma^{n} \\
K_{2}(t, \sigma)=\frac{t^{q n} \sigma^{-n}\left(1-\sigma^{-q n}\right)-\sigma^{-q n}\left(\sigma^{-n}-\sigma^{n}\right)}{1+\sigma^{-q n}}-t^{(q+1) n}+t^{n}-\sigma^{n} \\
K_{3}(t, \sigma)=\frac{t^{n} \sigma^{-q n}\left(\sigma^{2 n}-1\right)\left(t^{(q-1) n} \sigma^{-(q-1) n}-1\right)}{1+\sigma^{-2(q-1) n}}+t^{n}-\sigma^{n}
\end{array}\right.
$$

An analysis of the kernels $A_{j}(t, z)$ and $K_{j}(t, \sigma), j=1,2,3$ from (4.2) and (4.6) yields (2.3). 
To prove that the result is sharp, first we consider the point $z^{*}=\rho^{1+q}$ and the corresponding function $\hat{f}(z)=(z-\rho)^{-1}$. A direct computation from (3.1), (2.2) and (4.1) for $f=\hat{f}$ shows that

$$
R_{n+m, n}^{*}(z, \hat{f})-r_{n+m, n}(z, \hat{f})=\sum_{j=1}^{3} \frac{A_{j}(\rho, z) K_{j}(\rho, \sigma)}{\left(z^{n}-\sigma^{n}\right)(z-\rho)\left(\rho^{q^{n}}-1\right)}
$$

If $\sigma>\rho^{1+q}$, we get after some simple calculations

$$
\lim _{n \rightarrow \infty}\left\{R_{n+m, n}^{*}\left(\rho^{1+q}, \hat{f}\right)-r_{n+m, n}\left(\rho^{1+q}, \hat{f}\right)\right\}=\frac{\rho^{q}}{\rho^{q}-1}
$$

whereas for $\sigma=\rho^{1+q}$, the sequence (4.7) is undefined for infinitely many $n$ 's for $z=\rho^{1+q} \omega_{0}$ where $\omega_{0}$ is any primitive root of unity.

REMARK 4.1: If we fix $m=-1$ and $\sigma \rightarrow \infty$ in Theorem 2.1, we get a result of Rivlin $[1$, Theorem 1] in the special case when $m(n)=q n$.

REMARK 4.2: According to Remark 3.1, Saff-Sharma's result [2, Theorem 2.3] for the special case $m=-1$ can be retrieved from Theorem 2.1.

\section{REFERENCES}

[1] T.J. Rivlin, 'On Walsh Equiconvergence', J. Approx. Theory 36 (1982), 334-345.

[2] E.B. Saff and A. Sharma, 'On equiconvergence of certain sequence of rational Interpolants', $R a$ tional Approximation and Interpolation Lecture Notes in Math 1105 (Springer, 1984).

[3] J.L. Walsh, 'Interpolation and Approximation by Rational Functions in the complex Domain', Colloq. Publ. 5th ed., Amer. Math. Soc. (1969).

Centre for Advanced Studies in Pure and Applied Mathematics Bahauddin Zakariya University Multan, Pakistan. 